\title{
Effect on Microbial Growth of a New Skin Protectant Formulation
}

\author{
Joseph Stoffel ${ }^{1}$ and Stéphanie F. Bernatchez ${ }^{2, *}$ \\ ${ }^{1} 3 \mathrm{M}$ Critical \& Chronic Care Solutions Division, St. Paul, Minnesota. \\ ${ }^{2} 3 \mathrm{M}$ Health Care Clinical Operations, St. Paul, Minnesota.
}

Objective: Evaluate the effect of a new investigational skin protectant formulation on the growth of various microorganisms in vitro.

Approach: An in vitro laboratory assay with various species of gram-positive bacteria, gram-negative bacteria, and yeast grown on agar plates was used to verify that a new investigational product used for the management of incontinence-associated dermatitis (IAD) does not support microbial growth.

Results: The investigational product did not support the growth of all organisms tested for $48 \mathrm{~h}$ in these assays. The results demonstrate the barrier properties of this investigational formulation against bacteria and yeast that are relevant to incontinent patients.

Innovation: IAD accompanied by skin damage is difficult to manage with currently available products. A new skin protectant that can be applied as a liquid and polymerizes into a breathable film in situ even in the presence of exudate (as shown previously) has been developed and tested to ensure that it does not support microbial growth.

Conclusion: This work verifies that this new product does not support microbial growth in vitro using organisms relevant for the intended application.

Keywords: skin protectant, incontinence-associated dermatitis, microbial growth, in vitro

\section{INTRODUCTION}

INCONTINENCE-ASSOCIATED dermatitis (IAD) is a skin condition caused by the effects of urine and stool on the skin. Exposure to moisture and irritants can alter the skin $\mathrm{pH}$ and normal flora, and increase its susceptibility to damage from friction. ${ }^{1,2}$ Secondary infections often occur in IAD patients, given the susceptibility of moist and warm denuded skin; in fact, IAD is associated with an increased risk of localized cutaneous infection. ${ }^{3}$ The most common organisms are Candida albicans, from the gastrointestinal tract, and Staphylococcus species, from the perineal skin. ${ }^{4}$ The treatment of IAD focuses on three main goals: (1) removing irritants from the skin and protecting it from further exposure to urine or stool, (2) eradicating cutaneous infection such as candidiasis, and (3) alleviating, containing, or diverting incontinence-related urine or stool. ${ }^{3}$ Products used to manage IAD should

(c) Joseph Stoffel and Stéphanie F. Bernatchez, 2017; Published by Mary Ann Liebert, Inc. This Open Access article is distributed under the terms of the Creative Commons Attribution Noncommercial License (http://creativecommons.org/licenses/by-nc/4.0/) which permits any noncommercial use, distribution, and reproduction in any medium, provided the original author(s) and the source are credited.

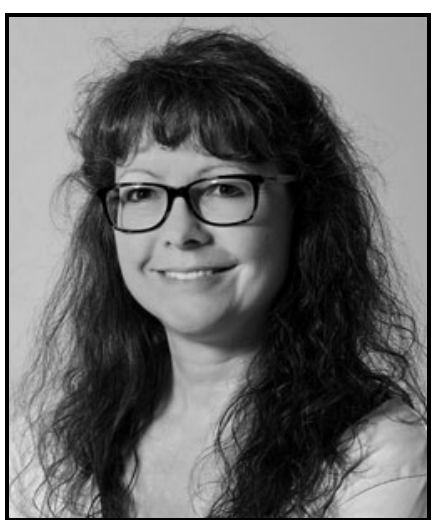

Stéphanie F. Bernatchez, PhD

Submitted for publication September 2, 2016 Accepted in revised form September 27, 2016.

*Correspondence: $3 \mathrm{M}$ Health Care Clinical Operations, 3M Center Bldg 270-3A-04, St. Paul, MN 55144-1000

(e-mail: sfbernatchez@mmm.com). 
not promote the growth of microorganisms, and ideally form a barrier to prevent them from penetrating the weakened skin.

A new skin protectant was developed to manage moderate to severe IAD. It consists of a customized acrylic polymer, combined with 2-octyl cyanoacrylate. Cyanoacrylates are generally recognized as having antimicrobial properties. ${ }^{5}$ The formulation combines a cyanoacrylate with a polymer in a novel chemistry, and we wanted to verify that the property of not supporting microbial growth was preserved. The purpose of this study was therefore to evaluate the effect of the new skin protectant on the growth of various microorganisms in an in vitro assay.

\section{CLINICAL PROBLEM ADDRESSED}

The skin denudement observed in severe cases of IAD is particularly challenging to manage because it often presents as multiple small open areas of irregular shape over a contoured body surface. These open areas are exudative and standard barrier film products, ointments, and even many pastes do not adhere to the wet weeping surface. In cases of frequent or continuous incontinence, the damaged skin is constantly exposed to moisture and irritants such as urine and/or feces. The skin is at a high risk for further breakdown and maceration. ${ }^{6}$ The investigational product described herein is applied as a liquid to effectively cover the desired area and conform to the topography. It polymerizes in situ into a film within $\sim 30 \mathrm{~s}$ even in the presence of exudate as previously shown in an animal model. ${ }^{7}$ This film is breathable and prevents irritants from reaching the skin surface. It remains intact even under conditions of continuous or repeated exposure. It provides an easy-to-clean surface after incontinence episodes and does not need to be reapplied each time. The results presented here demonstrate that in an in vitro assay, it does not support the growth of microorganisms relevant to incontinence patients.

\section{MATERIALS AND METHODS \\ Description of new formulation}

The investigational product is a solution based on a patented acrylate chemistry, which forms a durable, transparent elastomeric barrier upon application to skin. A customized acrylic polymer, combined with 2-octyl cyanoacrylate, creates the film structure. The film formers are delivered from a well-tolerated solvent. Prior testing done includes cytotoxicity, irritation, sensitization, genotoxicity, and systemic toxicity based on the criteria of expected use ( $>30$ days in contact with a breached skin barrier) and guidance covering the biological evaluation of medical devices outlined in EN ISO 10993-1:2009. The results (not shown) supported a conclusion that the product is safe for its intended use. Additional testing in three animal models has also been performed. ${ }^{7}$

\section{Microbial growth inhibition assay}

In this study, the formulation was tested by forming a film covering a partial area of the agar in Petri dishes and seeding the agar with various microorganisms (10 bacterial species and 3 yeasts). Figure 1 illustrates the deposition of the film on the agar plates, and Table 1 lists the various organisms tested. The organisms were purchased as individual singleuse KWIK-STIK ${ }^{\mathrm{TM}}$ devices from Microbiologics, Inc. (St. Cloud, MN), except for Staphylococcus aureus USA100 (clinical isolate from a previous study) and Pseudomonas aeruginosa PA-01 (gift from Dr. Gregory Schultz, University of Florida).

Sterile tryptic soy agar (TSA) and Sabouraud's dextrose agar (SDA) (Difco; Becton, Dickinson and Company, Sparks, MD) plates were poured in $90 \times 15 \mathrm{~mm}$ polystyrene Petri plates and allowed to solidify. Plates were inverted and stored at room temperature for $24 \mathrm{~h}$.

Pure culture stocks of all microbes were streaked onto TSA for bacteria and SDA for yeasts and grown overnight at $35^{\circ} \mathrm{C}$. Broth cultures were prepared by
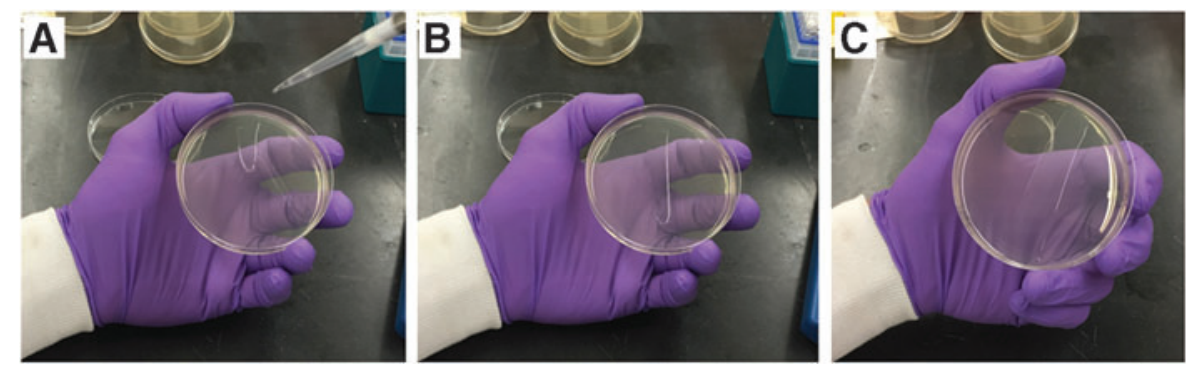

Figure 1. Application of investigational skin protectant to agar plates. (A) A pipette is used to deposit $100 \mu \mathrm{L}$ of the investigational product in one spot at the edge of the agar plate. (B) The plate is tilted vertically to have the drop spread linearly across the plate. (C) The plate is tilted horizontally to have the solution spread to form a broader line. 
Table 1. Microorganisms tested for growth inhibition by the investigational product

\begin{tabular}{ll}
\hline Microorganism & \multicolumn{1}{c}{ Comments } \\
\hline $\begin{array}{l}\text { Gram-positive bacteria } \\
\text { Staphylococcus aureus USA100 } \\
\text { (clinical isolate from previous study) }\end{array}$ & MRSA, clinical isolate \\
S. aureus ATCC 33592 & MRSA \\
S. aureus BAA-44 & Multidrug resistant \\
S. epidermidis ATCC 35984 & Biofilm former \\
Enterococcus faecalis ATCC 51299 & VRE \\
E. faecium ATCC 700221 & VRE \\
Gram-negative bacteria & \\
Pseudomonas aeruginosa BAA-2108 & Multidrug resistant \\
P. aeruginosa PA-01 (from Dr. Gregory & Biofilm former \\
$\quad$ Schultz, University of Florida) & \\
Acinetobacter baumannii BAA-1605 & Multidrug resistant \\
Escherichia coli BAA-2469 & Multidrug resistant, NDM-1 \\
Klebsiella pneumoniae BAA-2146 & Multidrug resistant, NDM-1 \\
Enterobacter cloacae ATCC 23355 & Drug resistant \\
Serratia marcescens ATCC 14756 & Reference strain \\
Yeast & \\
Candida albicans ATCC 10231 & Reference strain \\
C. tropicalis ATCC 9968 & Reference strain \\
C. glabrata ATCC 15545 & Reference strain \\
\hline
\end{tabular}

NDM-1, New Delhi metallo-beta-lactamase multidrug-resistant strain; MRSA, methicillin-resistant Staphylococcus aureus; VRE, vancomycinresistant Enterococcus.

inoculating $5 \mathrm{~mL}$ of TSB with an isolated colony from the stock plates and incubated overnight at $35^{\circ} \mathrm{C}$. The broth cultures were centrifuged to a pellet and resuspended in phosphate-buffered water (PBW). Suspensions of each organism were prepared using McFarland turbidity standards to a density of $\sim 10^{8}$ colony forming unit $(\mathrm{cfu}) / \mathrm{mL}$ in PBW. Suspensions were diluted 1:1000 in PBW to a final concentration of $\sim 10^{5} \mathrm{cfu} / \mathrm{mL}$.

The investigational product ( $100 \mu \mathrm{L}$ of solution) was pipetted and transferred to the top edge agar surface of the Petri plate while holding it at a slight angle. After the solution ran to the opposite edge, the plate was tilted to the right, which allowed the film to spread, creating an $\sim 2 \mathrm{~cm}$ width band down the center of the plate (Fig. 1). Plates were allowed to dry in a biological safety cabinet with the lids slightly ajar for $15 \mathrm{~min}$ (normal dry time on skin is about $30 \mathrm{~s}$; additional time was allowed in this experiment to ensure full polymerization in this different environment). Two milliliters of the diluted bacteria $\left(10^{5} \mathrm{cfu} / \mathrm{mL}\right.$ suspension) was pipetted onto the film. The plate was rotated until the entire surface had been contacted by the bacterial suspension. The plates were allowed to stand for $60 \mathrm{~s}$, after which the remaining suspension was decanted from the plate. The plates were left in a biological safety cabinet with the lids slightly ajar for $10 \mathrm{~min}$ to allow any pooled inoculum to dissipate. The plates were then incubated inverted for $48 \mathrm{~h}$ at $35^{\circ} \mathrm{C}$. Plates were observed at 24 and $48 \mathrm{~h}$ for growth. Every organism was run in duplicate.

Results were recorded and photos of each plate were taken. A successful result was indicated by a clear zone with no microbial growth where the investigational product was applied.

\section{RESULTS}

Every plate displayed a clear zone (no microbial growth) where the investigational product was applied, and a full microbial lawn on the rest of the plate, confirming viability and growth of the organism. Figure 2 shows control plates and Figure 3 shows representative samples for each of the various microorganisms.

\section{DISCUSSION}

Our results show that the new investigational skin protectant did not support the growth of several gram-positive organisms, gram-negative organisms, and yeasts in a laboratory assay over a period of $48 \mathrm{~h}$.

As mentioned previously, various other formulations containing cyanoacrylates have been shown to provide a barrier to bacterial penetration. Bhende et $a l .{ }^{8}$ used a laboratory assay with agar plates containing a $\mathrm{pH}$ indicator to indicate actively growing bacteria and showed that the cyanoacrylate tissue adhesive formulation tested (Dermabond ${ }^{\circledR}$, 2-octylcyanoacrylate) was an effective microbial

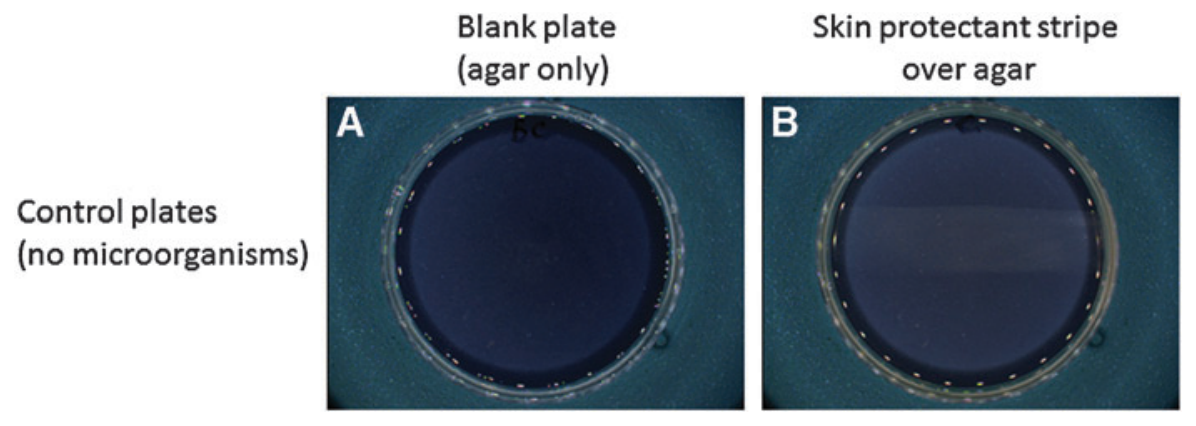

Figure 2. (A) Control plate with agar only. (B) Plate with a strip of skin protectant (a low level of haziness is inherent to the product). 
A

Organism
Methicillin-resistant
Staphylococcus aureus
USA100 (clinical isolate)

Methicillin-resistant Staphylococcus aureus ATCC 33592

Methicillin-resistant Staphylococcus aureus BAA-44

taphylococcus epidermidis ATCC 35984

Enterococcus faecalis. ATCC 51299

Enterococcus faecium ATCC 700221
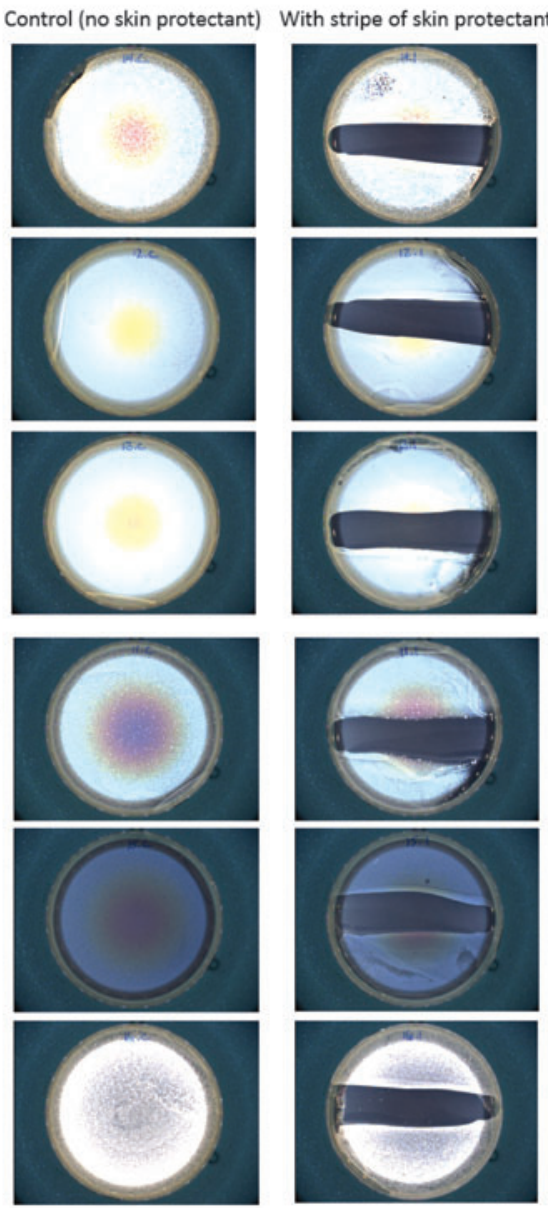

C

Organism
Candida albicans
ATCC 10231

ATCC 10231
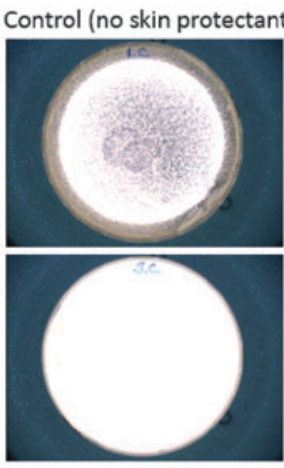

Candida tropicalis ATCC 9968
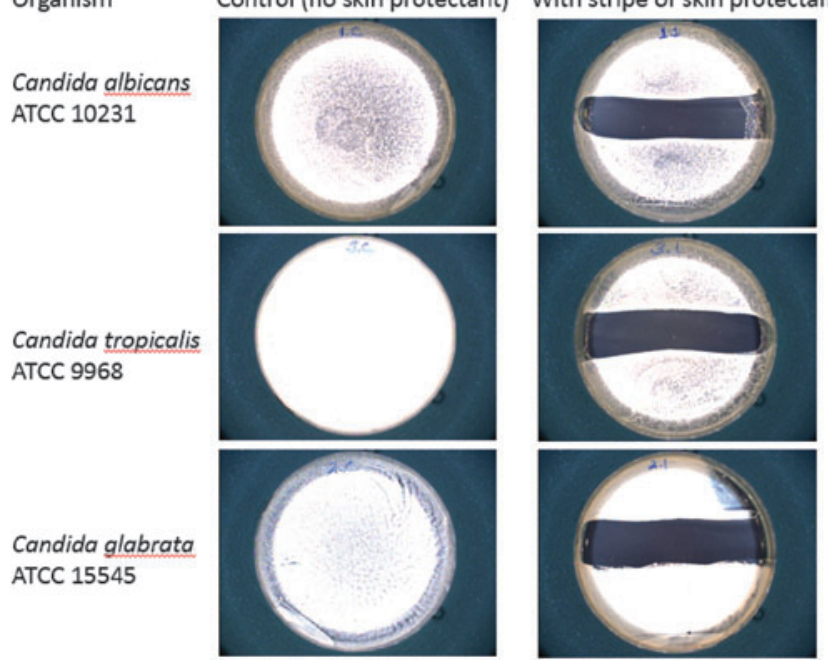

B

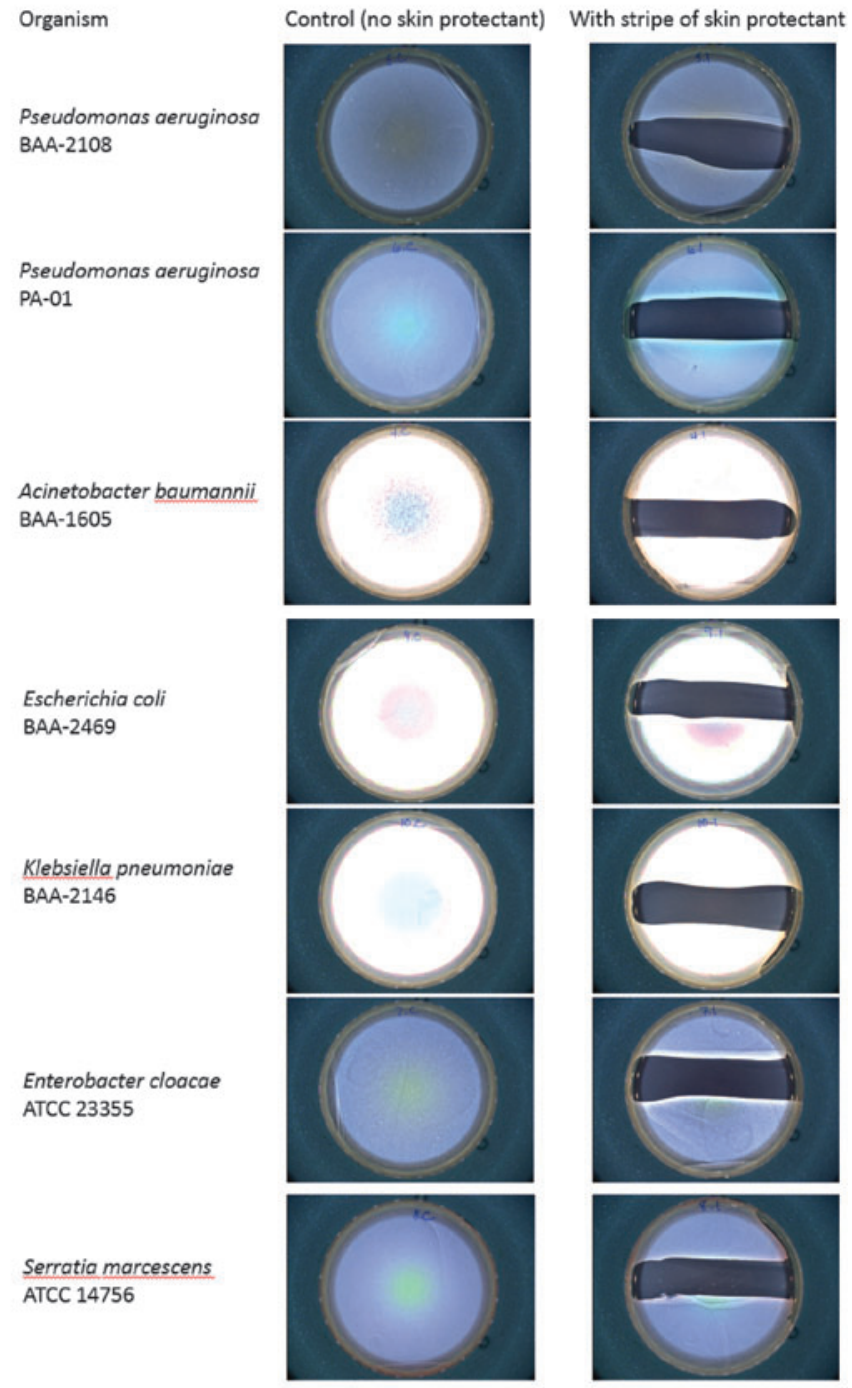

Control (no skin protectant) With stripe of skin protectant

Figure 3. Agar plates after a 48-h incubation. Left. Plate seeded with microorganism. Right. Plate covered with a strip of investigational formulation and then seeded with microorganism. (A) Gram-positive organisms. (B) Gram-negative organisms. (C) Yeasts. 
barrier. As they pointed out, this type of test is much more sensitive than an animal infection model, since penetration of one organism $(1 \mathrm{cfu})$ would theoretically lead to a positive test, whereas logarithmically higher numbers of organisms would be required to establish an infection in an in vivo model due to the animals' inherent infection resistance/immune response. Narang et al. ${ }^{9}$ obtained similar results while performing their experiment in two different ways (also using Dermabond), either polymerizing the cyanoacrylate film directly on the agar as we did or using a preformed film in case the polymerization affected the agar (which it did not). Another agar plate method intended for a different application was used by Simonova et al. ${ }^{10}$ to test 2 cyanoacrylate adhesives (Dermabond, 2-octylcyanoacrylate; and Histoacryl $^{\circledR}$, butylcyanoacrylate) for their ability to prevent the migration of microbes inoculated around catheters inserted in the agar at a $30^{\circ}$ angle. Their results showed no visible growth under the cyanoacrylate and no penetration along the catheter tunnel. Romero et al. ${ }^{11}$ have shown zones of inhibition in vitro for different cyanoacrylate chemistries (Superbonder ${ }^{\circledR}$, ethyl-cyanoacrylate; and Histoacryl, butylcyanoacrylate) for gram-positive bacteria, but not gram negatives. They hypothesize that the antibacterial effects of cyanoacrylate are greater in gram-positive bacteria than in gram-negative bacteria, possibly because the latter is protected by an outer carbohydrate capsule. Similar zone of inhibition results were also described by Friedman et al. ${ }^{12}$ using Neucrylate ${ }^{\mathrm{TM}}$ (1-hexyl-2-cyanoacrylate). On the contrary, Olson et al. ${ }^{13}$ showed in vitro adhesion of S. epidermidis to VetBond ${ }^{\mathrm{TM}}$ (n-butyl-2cyanoacrylate), followed by biofilm formation. Their method involved the circulation of medium containing bacteria with a peristaltic pump, which is different than the inoculation on Petri plates. This is, to our knowledge, the only publication describing actual bacterial growth on a cyanoacrylate, and our own results with a different strain of this biofilm former organism are contrary to those reported in that article. Matsumoto et al. ${ }^{14}$ have described the absence of bacteriostatic and bactericidal effects in vitro for individual monomers of n-butyl, isobutyl, and methyl-2 cyanoacrylate. Finally, Jang et al. ${ }^{15}$ have shown no in vitro antibacterial activity of octylcyanoacrylate against clinical strains of $P$. aeruginosa isolated from otitis media patients, which is consistent with other results discussed above regarding gram-negative bacteria. We believe that it is difficult to strictly compare in vitro results from different studies because the bacterial strains used were different, the inoculates were different, the volumes and chemical compositions of cyanoacry- lates used were different, and mostly because the cyanoacrylate we describe here is a novel formulation combining other ingredients. In addition to these in vitro methods, pig models of partial-thickness wounds were used in which a cyanoacrylate-based formulation was applied to wounds either before (barrier study) or after (antimicrobial study) a bacterial inoculum challenge. The formulation protected wounds from external pathogens (compared to a first aid dressing and air exposed) and also created an environment that was not conducive to the growth of $S$. aureus and $P$. aeruginosa and was able to reduce the number of organisms recovered from the wounds compared to the other treatments. ${ }^{16}$ Another study has been done to look at the effect of using a cyanoacrylate product (InteguSeal ${ }^{\circledR}, n$-butyl cyanoacrylate) as a preoperative sealant in trauma surgery patients and found that this intervention reduced microbial contamination on sutures during surgery, without, however, changing overall wound contamination. ${ }^{17}$ Finally, Dohmen ${ }^{18}$ reviewed the literature on the impact of microbial skin sealants to prevent surgical site infections (SSIs) and concluded that a cyanoacrylate skin sealant can prohibit endogenous bacteria migration (by immobilizing any bacteria having survived the skin antiseptics) and actively reduce bacterial growth, making it an attractive option to reduce SSIs.

The ability of cyanoacrylate to immobilize bacteria could prove to be a helpful strategy to help prevent infections on wounded skin. Recent research has shown that many wound dressings dramatically reduce the antimicrobial activity of local wound antiseptics and wound irrigating agents (in vitro data). ${ }^{19}$ If the antiseptic properties of wound cleaning agents are also compromised during their clinical use because of possible negative interaction or interference with other wound care products, then an additional component of treatment, including bacterial immobilization, could potentially be beneficial to successfully fight infection.

This study has limitations. Agar is different from skin and may not reflect the clinical situation. The mechanism of action has not been addressed; however, one could speculate that since adherence of bacteria or fungi to target tissue is an important first step in infection, a cyanoacrylate product bound to skin could likely prevent infection by inhibiting the adhesion of microorganisms to the tissue and preventing deeper penetration in the tissue. Joost et al. ${ }^{20}$ have shown that the extracellular adherence protein of $S$. aureus is more highly expressed in deep chronic wounds than in superficial wounds, supporting the hypothesis that adherence could contribute to the development of chronic infection. Microbial attachment is also recognized as the first 
step in the establishment of biofilms, and chronic wounds have been shown to harbor biofilms. ${ }^{21,22}$

The present in vitro results confirm that the new formulation does not support the growth of the microorganisms tested on its surface nor does it allow them to penetrate through it to reach the nutrient layer below. When combined with the other information gathered thus far on this investigational product, that is, biocompatibility testing (results not shown), animal testing, ${ }^{7}$ and clinical testing (manuscript accepted for publication), this novel skin protectant shows promise for the management of IAD.

\section{INNOVATION}

The innovative property of the investigational skin protectant presented here is that it can form a durable, long-lasting polymeric film in situ even in the presence of exudate. ${ }^{7}$ This film is breathable and capable of preventing irritants from reaching the skin surface. This study demonstrates that the film also does not support the growth of microorganisms on it and prevents them from penetrating through it to reach the nutrient layer below in an in vitro microbial culture assay.

\section{ACKNOWLEDGMENTS AND FUNDING SOURCES}

This work was done in the laboratories of 3M.

\section{AUTHOR DISCLOSURE AND GHOSTWRITING}

J.S. and S.F.B. are employees of 3M. Both authors contributed to writing this article and no ghostwriters were used to write this article.

\section{ABOUT THE AUTHORS}

Joseph J. Stoffel, BS, in Microbiology from the University of Wisconsin-La Crosse. He currently resides in Minnesota and works for $3 \mathrm{M}$. His work at $3 \mathrm{M}$ has included research and development in the area of advanced wound care, biofilm prevention, food safety, diagnostics, and inhalation drug delivery using in vitro and in vivo assays, clinical research, and analytical chemistry. Stéphanie F. Bernatchez, PhD, in Interdisciplinary Sciences from the University of Geneva, Switzerland. She currently resides in Minnesota and works for 3M. Her work at $3 \mathrm{M}$ has included research and development in the area of advanced wound care using in vitro and in vivo assays, as well as clinical research work.

\section{REFERENCES}

1. Chatham N, Carls C. How to manage incontinence-associated dermatitis. Wound Care Advisor 2012;1:7-10.

2. Farage MA, Miller KW, Berardesca E, Maibach HI Incontinence in the aged: contact dermatitis and other cutaneous consequences. Contact Dermatitis 2007:57:211-217.

3. Gray M. Optimal management of incontinenceassociated dermatitis in the elderly. Am J Clin Dermatol 2010;11:201-210.

4. Beeckman D, Schoonhoven L, Verhaeghe S, Heyneman A, Defloor T. Prevention and treatment of incontinence-associated dermatitis: literature review. J Adv Nurs 2009;65:1141-1154.

5. Burns B. Polycyanoacrylates. In: Encyclopedia of Polymer Science and Technology. John Wiley \& Sons, Inc., 2016:1-27. http://onlinelibrary.wiley .com/doi/10.1002/0471440264.pst256.pub2/abstract;
jsessionid=D851C72B9A16B85582D64CD42DEAC CE3.f02t01 (last accessed November 1, 2016).

6. Beeckman D, Campbell J, Campbell K, et al. Proceedings of the Global IAD Expert Panel. Incontinence-associated dermatitis: moving prevention forward. Wounds International. www .woundsinternational.com, 2015:1-24.

7. Been RA, Bernatchez SF, Conrad-Vlasak DM, Asmus RA, Ekholm BP, Parks PJ. In vivo methods to evaluate a new skin protectant for loss of skin integrity. Wound Repair Regen 2016;24:851859

8. Bhende S, Rothenburger S, Spangler DJ, Dito M. In vitro assessment of microbial barrier properties of Dermabond topical skin adhesive. Surg Infect (Larchmt) 2002;3:251-257.

9. Narang U, Mainwaring L, Spath G, Barefoot J. Invitro analysis for microbial barrier properties of 2- octyl cyanoacrylate-derived wound treatment films. J Cutan Med Surg 2003;7:13-19.

10. Simonova G, Rickard CM, Dunster KR, Smyth DJ, McMillan D, Fraser JF. Cyanoacrylate tissue adhesives-effective securement technique for intravascular catheters: in vitro testing of safety and feasibility. Anaesth Intensive Care 2012;40: 460-466

11. Romero IL, Malta JBNS, Silva CB, Mimica LMJ, Soong KH, Hida RY. Antibacterial properties of cyanoacrylate tissue adhesive: does the polymerization reaction play a role? Indian $\mathrm{J}$ Ophthalmol (Poona City) 2009;57:341-344.

12. Friedman P, Casillas V, Kerber CW. 1-Hexyl-2cyanoacrylate compound (Neucrylate) bactericidal properties. J Neurointerv Surg 2012:4:379-384.

13. Olson ME, Ruseska I, Costerton JW. Colonization of n-butyl-2-cyanoacrylate tissue adhesive by 
Staphylococcus epidermidis. J Biomed Mater Res 2004;22:485-495

14. Matsumoto T, Dobek AS, Pani KC, Kovaric JJ, Hamit HF. Bacteriological study of cyanoacrylate tissue adhesives. Arch Surg 1968;97:527530.

15. Jang $\mathrm{CH}$, Park H, Cho YB, Choi CH, Song C. Antibacterial effect of octylcyanoacrylate against ciprofloxacin-resistant Pseudomonas aeruginosa isolates from patients with chronic suppurative otitis media. In Vivo 2009;23:183-186.

16. Mertz PM, Davis SC, Cazzaniga AL, Drosou A, Eaglstein WH. Barrier and antibacterial properties of 2-octyl cyanoacrylate-derived wound treatment films. J Cutan Med Surg 2003;7:1-6.

17. Daeschlein G, Napp M, Assadian 0, et al. Influence of preoperative skin sealing with cyanoac- rylate on microbial contamination of surgical wounds following trauma surgery: a prospective, blinded, controlled observational study. Int J Infect Dis $2014 ; 29: 274-278$.

18. Dohmen PM. Impact of antimicrobial skin sealants on surgical site infections. Surg Infect (Larchmt) 2014;15:368-371.

19. Hirsch T, Limoochi-Deli S, Lahmer A, et al. Antimicrobial activity of clinically used antiseptics and wound irrigating agents in combination with wound dressings. Plast Reconstr Surg 2011;127: 1539-1545.

20. Joost I, Blass D, Burian M, et al. Transcription analysis of the extracellular adherence protein from Staphylococcus aureus in authentic human infection and in vitro. J Infect Dis 2009;199: 1471-1478.
21. James GA, Swogger $E$, Wolcott $R$, et al. Biofilms in chronic wounds. Wound Repair Regen 2008;16: 37-44.

22. Bjarnsholt T, Kirketerp-Moller K, Jensen PO, et al. Why chronic wounds will not heal: a novel hypothesis. Wound Rep Reg 2008;16:2-10.

\section{Abbreviations and Acronyms}

$I A D=$ incontinence-associated dermatitis MRSA = methicillin-resistant Staphylococcus aureus

PBW $=$ phosphate-buffered water

SDA $=$ Sabouraud's dextrose agar

SSIs = surgical site infections

TSA $=$ tryptic soy agar

VRE = vancomycin-resistant Enterococcus 